\title{
JIHADI GROUPS, NUCLEAR PAKISTAN, AND THE NEW GREAT GAME
}

M. Ehsan Ahrari

August 2001 